\title{
Analysis of dynamic mechanical, low-velocity impact and compression after impact behaviour of benzoyl treated sugar palm/glass/epoxy composites
}

\begin{abstract}
The objective of this work is to investigate the effect of glass fibre addition and benzoylation treatment on the dynamic mechanical, impact and post-impact properties of sugar palm composites. The hybrid composites were prepared using hand lay-up method with different volume fractions of sugar palm fibre and glass fibre. Dynamic mechanical analysis (DMA) results show that EP/30TSPF/70GF composites exhibited the highest loss and storage modulus compared to EP/UTSPF composites. For the low-velocity impact testing, the experimental results show that the impact energy displayed an excellent relationship with the impact response. The ultrasonic $\mathrm{C}$-scan images show that the damaged area is increasing as the impact energy increasing. From the compression after impact (CAI) studies, it could be resolved that the impact damage exhibited outstanding connection with the impact response and compressive strength of the EP/30TSPF/70GF composite decreased as the impact energy increasing. Overall, it can be concluded that the benzoylation treatment and glass fibre addition on the sugar palm composites enhances the dynamic mechanical, impact and postimpact properties of sugar palm composites.
\end{abstract}

Keyword: Hybrid composites; Impact behaviour; Dynamic mechanical thermal analysis (DMTA); Ultrasonics 